\title{
SEPARATION PROPERTIES AT $p$ FOR THE TOPOLOGICAL CATEGORY OF CLOSURE SPACES
}

\section{DENIZ TOKAT}

Department of Mathematics

Nevşehir Haci Bektaş Veli University

Nevşehir

Turkey

e-mail: dtokat@nevsehir.edu.tr

\begin{abstract}
Each of the separation properties $T_{i}, i=0,1,2$ at a point $p$ is given in the topological category of closure spaces. Furthermore, the relationships between these properties of the category are studied.
\end{abstract}

\section{Introduction}

Despite the fact that closure operators had been used in calculus first ([27] and [29]), they have been used in other fields of mathematics such as logic ([24] and [30]), algebra ([12], [13], and [28]), and topology ([26] and [14]).

In 1940, Birkhoff observed that the collection of closed sets of a closure space forms a complete lattice [13]. Since his work, the interrelation between closures and complete lattices has been investigated by many authors and a general treatment of this subject can 
be found in [21]. Another motivation for considering closures is Birkhoff's work on association of closures to binary relations in his book [13]. By using similar ideas, Aumann worked on contact relations with application to social sciences [4] or Ganter and Wille worked on formal contexts with application to data analysis and knowledge representation [22].

In recent years, closure operators are used in quantum logic and representation theory of physical systems [2], [3].

A closure space $(X, \mathcal{C})$ is a pair, where $X$ is a set and $\mathcal{C}$ is a subset of the power set $P(X)$ satisfying the conditions that $X$ and $\emptyset$ belong to $\mathcal{C}$ and that $\mathcal{C}$ is closed for arbitrary unions. A function $f:(X, \mathcal{C}) \rightarrow(Y, \mathcal{D})$ between closure spaces $(X, \mathcal{C})$ and $(Y, \mathcal{D})$ is said to be continuous if $f^{-1}(D) \in \mathcal{C}$ whenever $D \in \mathcal{D}$. Cls is the construct with closure spaces as objects and continuous maps as morphisms [17].

Another isomorphic description is obtained by means of a closure operator [13]. The closure operation $c l: P(X) \rightarrow P(X)$ associated with a closure space $(X, \mathcal{C})$ is defined in the usual way by $x \in \operatorname{cl} A \Leftrightarrow(\forall C \in \mathcal{C}$ $: x \in C \Rightarrow C \cap A \neq \emptyset$ ), where $A \subset X$ and $x \in X$. This closure need not be finitely additive, but it does satisfy the conditions $\operatorname{cl\emptyset }=\emptyset,(A \subset B \Rightarrow$ $c l A \subset \operatorname{clB}), A \subset \operatorname{cl} A$, and $\operatorname{cl}(\operatorname{cl} A)=\operatorname{cl} A$ whenever $A$ and $B$ are subsets of $X$. Continuity is then characterized in the usual way [17].

Finally, closure spaces can also be equivalently described by means of neighbourhood collections of the points. These neighbourhood collections satisfy the usual axioms, except for the fact that the collections need not be filters. So in a closure space $(X, \mathcal{C})$ the neighbourhood collection of a point $x$ is a non empty stack (in the sense that with every $V \in \mathcal{N}(x)$ also every $W$ with $V \subset W$ belongs to $\mathcal{N}(x)$ ), where every $V \in \mathcal{N}$ contains $x$ and $\mathcal{N}(x)$ satisfies the open kernel condition [17]. 
The notions of "closedness" and "strong closedness" in set based topological categories are introduced by Baran [5], [6] it is shown in [8] that these notions form an appropriate closure operator in the sense of Dikranjan and Giuli [18] in some well-known topological categories. Moreover, various generalizations of each of $T_{i}, i=0,1,2,3,4$ separation properties for an arbitrary topological category over Set, the category of sets are given and the relationship among various forms of each of these notions are investigated by Baran in [5], [7], [9], and [10].

In the last decade, complete objects in the category of $T_{0}$ closure spaces is characterized by Deses et al. [17] and a cartesian closed topological hull and quasitopos hull of the construct Cls of closure spaces is constructed by Claes et al. (see [15] and [16]).

Recently, in [11], the characterization of each of the various notions of connected and $T_{i}, i=0,1,2$ closure spaces are given and examined how these generalizations are related.

The main goal of this paper is to study each of the separation properties $T_{i}, i=0,1,2$ at a point $p$ in the topological category of closure spaces. In addition, we will investigate the relationships between separation properties at a point $p$ in this category.

\section{Preliminaries}

Recall, [1], that a functor $U: \mathcal{E} \rightarrow$ Set is said to be topological, or that $\mathcal{E}$ is a topological category over Set, the category of sets, if $U$ is concrete (i.e., faithful and amnestic (i.e., if $U(f)=i d$ and $f$ is an isomorphism, then $f=i d)$ ), has small (i.e., set) fibers, and is such that every $U$-source has an initial lift or, equivalently, is such that each $U$ sink has a final lift.

The categorical terminology is that of [1]. 
A closure space $(X, \mathcal{C})$ is a pair, where $X$ is a set and $\mathcal{C}$ is a subset of the power set $P(X)$ satisfying the conditions that $X$ and $\emptyset$ belong to $\mathcal{C}$ and that $\mathcal{C}$ is closed for arbitrary unions. A function $f:(X, \mathcal{C}) \rightarrow(Y, \mathcal{D})$ between closure spaces $(X, \mathcal{C})$ and $(Y, \mathcal{D})$ is said to be continuous if $f^{-1}(D) \in \mathcal{C}$ whenever $D \in \mathcal{D}$. Cls is the category with closure spaces as objects and continuous maps as morphisms [17].

Cls is a topological category [19] and Top, the category of topological spaces, is embedded in Cls as a full bicoreflective subconstruct [15].

Note that a source $\left\{f_{i}:(X, \mathcal{C}) \rightarrow\left(Y_{i}, \mathcal{C}_{i}\right), i \in I\right\}$ is initial in Cls iff $\mathcal{C}=\left\{U \subset X: U=\bigcup_{i \in I} f_{i}^{-1}\left(U_{i}\right), U_{i} \in \mathcal{C}_{i}\right\}$ [23].

Similarly, an epi sink $f_{i}:\left(Y_{i}, \mathcal{C}_{i}\right) \rightarrow(X, \mathcal{C})$ is final in Cls iff $\mathcal{C}=\left\{U \subset X: f_{i}^{-1}(U) \in \mathcal{C}_{i}\right.$, for all $\left.i \in I\right\}$.

In particular:

(1) The embeddings $f: X \rightarrow Y$ are the injective maps such that a subset of $\mathrm{X}$ is open iff it is inverse image by $f$ of an open set of $Y$.

(2) Let $\left\{\left(X_{i}, \mathcal{C}_{i}\right)\right\}$ be a collection of closure spaces and $X$ be the product of the sets $X_{i}$, i.e., $X=\prod_{i} X_{i}$. The product structure on $X$ is the class $\mathcal{C}=\left\{U \subset X: U=\bigcup_{i \in I} \pi_{i}^{-1}\left(U_{i}\right), U_{i} \in \mathcal{C}_{i}\right\}$

(3) $(X, \mathcal{C})$ is a discrete space $\mathcal{C}=P(X)$ and it is an indiscrete space $\mathcal{C}=\{X, \emptyset\}$.

\section{Separation Properties at $p$}

Let $B$ be a set and $p \in B$. Let $B \vee_{p} B$ be the wedge at $p$ [5], i.e., two disjoint copies of $B$ identified at $p$, or in other words, the pushout of $p: 1 \rightarrow B$ along itself (where 1 is a terminal object in Set). More 
precisely, if $i_{1}$ and $i_{2}: B \rightarrow B \vee_{p} B$ denote the inclusions of $B$ as the first and the second factor, respectively, the $i_{1} p=i_{2} p$ is a pushout diagram. A point $x$ in $B \vee_{p} B$ will be denoted by $x_{1}\left(x_{2}\right)$ if $x$ is in the first (resp., the second) component of $B \vee_{p} B$. Note that $p_{1}=p_{2}$.

The principle $p$-axis map $A_{p}: X \vee_{p} X \rightarrow X^{2}$ is defined by $A_{p}\left(x_{1}\right)=$ $(x, p)$ and $A_{p}\left(x_{2}\right)=(p, x)$. The skewed $p$-axis map $S_{p}: B \vee_{p} B \rightarrow B^{2}$ is given by $S_{p}\left(x_{1}\right)=(x, x)$ and $S_{p}\left(x_{2}\right)=(p, x)$. The fold map at $p$, $\nabla_{p}: B \vee_{p} B \rightarrow B$ is given by $\nabla_{p}\left(x_{i}\right)=x$ for $i=1,2$ [5] or [6].

Note that the maps $A_{p}, S_{p}$, and $\nabla_{p}$ are the unique maps arising from the above pushout diagram for which $A_{p} i_{1}=(i d, p): B \rightarrow B^{2}$, $A_{p} i_{2}=(p, i d): B \rightarrow B^{2}, S_{p} i_{1}=(i d, i d): B \rightarrow B^{2}, S_{p} i_{2}=(p, i d): B \rightarrow B^{2}$, and $\nabla_{p} i_{j}=i d, j=1,2$, respectively, where $i d: B \rightarrow B$ is the identity map and $p: B \rightarrow B$ is the constant map at $p$.

The infinite wedge product $\vee_{p}^{\infty} B$ is formed by taking countably many disjoint copies of $B$ and identifying them at $p$. Let $B^{\infty}=B \times B \times \ldots$ be the countable cartesian product of $B$. Define $A_{p}^{\infty}: \vee_{p}^{\infty} B \rightarrow B^{\infty}$ by $A_{p}^{\infty}\left(x_{i}\right)=(p, p, \ldots, x, p, p, \ldots)$, where $x_{i}$ is the $i$-th component of the infinite wedge and $x$ is in the $i$-th place in $(p, p, \ldots, x, p, p, \ldots)$ and $\nabla_{p}^{\infty}: \vee_{p}^{\infty} B \rightarrow B$ by $\vee_{p}^{\infty}\left(x_{i}\right)=x$ for all $i,[5]$ or [6].

Note, also, that the map $A_{p}^{\infty}$ is the unique map arising from the multiple pushout of $p: 1 \rightarrow B$ for which $A_{p}^{\infty} i_{j}=(p, p, p, \ldots, p, i d, p, \ldots)$ $: B \rightarrow B^{\infty}$, where the identity map, $i d$, is in the $j$-th place. 
Let $U: \mathcal{E} \rightarrow$ Set be topological and $X$ an object in $\mathcal{E}$ with $U(X)=B$. Let $M$ be a non-empty subset of $B$. We denote by $X / M$ the final lift of the epi $U$-sink $q: U(X)=B \rightarrow B / M=(B \backslash M) \cup\{*\}$, where $q$ is the epi map that is the identity on $B \backslash M$ and identifying $M$ with a point * [5].

Baran [5] introduced the definition of various separation properties for arbitrary topological category over sets. Moreover, he defined the notions of closed points, closed objects, and strongly closed objects.

Definition 3.1 (cf. [5] and [6]). Let $U: \mathcal{E} \rightarrow$ Set be a topological functor, $D$ is the discrete functor which is a left adjoint of $U, X$ an object in $\mathcal{E}$, and $p$ a point in $U X=B$.

(1) $X$ is $\bar{T}_{0}$ at $p$ iff the initial lift of the $U$-source $\left\{A_{p}: B \vee_{p} B \rightarrow\right.$ $U\left(X^{2}\right)=B^{2}$ and $\left.\nabla_{p}: B \vee_{p} B \rightarrow U D B=B\right\}$, is discrete.

(2) $X$ is $T_{0}^{\prime}$ at $p$ iff the initial lift of the $U$-source $\left\{i d: B \vee_{p} B \rightarrow U\right.$ $\left(X \vee_{p} X\right)=B \vee_{p} B$ and $\left.\nabla_{p}: B \vee_{p} B \rightarrow U D B=B\right\}$ is discrete, where $X \vee_{p} X$ is the wedge in $\mathcal{E}$ i.e. the final lift of the $U$-sink $\left\{i_{1}, i_{2}: U X=\right.$ $\left.B \rightarrow B \vee_{p} B\right\}$, where $i_{1}, i_{2}$ denote the canonical injections.

(3) $X$ is $T_{1}$ at $p$ iff the initial lift of the $U$-source $\left\{S_{p}: B \vee_{p} B \rightarrow U\right.$ $\left(X^{2}\right)=B^{2}$ and $\left.\nabla_{p}: B \vee_{p} B \rightarrow U D B=B\right\}$ is discrete.

(4) $X$ is $\operatorname{Pre} \bar{T}_{2}$ at $p$ iff the initial lift of the $U$-source $\left\{S_{p}: B \vee_{p} B \rightarrow\right.$ $\left.U\left(X^{2}\right)=B^{2}\right\}$ and the initial lift of the $U$-source $\left\{A_{p}: B \vee_{p} B \rightarrow U\left(X^{2}\right)\right.$ $\left.=B^{2}\right\}$ agree.

(5) $X$ is $\operatorname{PreT}_{2}^{\prime}$ at $p$ iff the initial lift of the $U$-source $\left\{S_{p}: B \vee_{p} B \rightarrow\right.$ $\left.U\left(X^{2}\right)=B^{2}\right\}$ and the final lift of the $U$-sink $\left\{i_{1}, i_{2}: U X=B \rightarrow B \vee_{p} B\right\}$ agree. 
(6) $X$ is $\bar{T}_{2}$ at $p$ iff $X$ is $\bar{T}_{0}$ at $p$ and $\operatorname{Pr} e \bar{T}_{2}$ at $p$.

(7) $X$ is $T_{2}^{\prime}$ at $p$ iff $X$ is $T_{0}^{\prime}$ at $p$ and $P r e T_{2}^{\prime}$ at $p$.

Theorem 3.2 (cf. [5]). For the category of topological spaces, we have

(1) $\bar{T}_{0}$ at $p$ is equivalent to $T_{0}^{\prime}$ at $p$ and they both reduce to the following (called $T_{0}$ at $p$ ): for each point $x$ distinct from $p$, there exists a neighbourhood of $x$ missing $p$ or there exists a neighbourhood of $p$ missing $x$.

(2) $\operatorname{Pre} \bar{T}_{2}$ at $p$ is equivalent to PreT $T_{2}^{\prime}$ at $p$ and they both reduce to the following (called Pre $T_{2}$ at $p$ ): for each point $x$ distinct from $p$, if the set $\{x, p\}$ is not indiscrete, then there exist disjoint neighbourhoods of $x$ and $p$.

(3) $\bar{T}_{2}$ at $p$ is equivalent to $T_{2}^{\prime}$ at $p$ and they both reduce to the following (called $T_{2}$ at $p$ ): for each point $x$ distinct from $p$, then there exist disjoint neighbourhoods of $x$ and $p$.

Theorem 3.3. Let $(X, \mathcal{C})$ be a closure space and $p \in X$. The space $X$ is $\bar{T}_{0}$ at $p$ iff $X$ is a singleton.

Proof. Assume that $(X, \mathcal{C})$ is $\bar{T}_{0}$ at $p$. Let $x \neq p$ and $x \in X$. Then $\left\{x_{1}\right\} \in P\left(X \vee_{p} X\right)$, where $P\left(X \vee_{p} X\right)$ is the discrete structure on $X \vee_{p} X$. Assume also that $\mathcal{C}^{2}$ is the product structure on $X^{2}$ and $W \in \mathcal{C}^{2}$, then we get $\nabla_{p}^{-1}(\{x\}) \cup A_{p}^{-1}(W)=\left\{x_{1}\right\}$. Since $A_{p}\left(x_{1}\right)=(x, p) \in W$ and $W \in \mathcal{C}^{2}$, we have $N, M \in \mathcal{C}$ such that $W=\pi_{1}^{-1}(N) \cup \pi_{2}^{-1}(M)=(N \times X)$ $\cup(X \times M)$. On the other hand, if $x \neq p$, then we find $\nabla_{p}^{-1}(\{x\})=\left\{x_{1}, x_{2}\right\}$. However, since we get $\left\{x_{1}\right\}=\nabla_{p}^{-1}(\{x\}) \cup A_{p}^{-1}(W)$ supset $\left\{x_{1}, x_{2}\right\}$, which is impossible. Hence, we get $X=\{p\}$.

The other side of the implication is trivial.

Theorem 3.4. Let $(X, \mathcal{C})$ be a closure space and $p \in X$. The space $X$ is $T_{0}^{\prime}$ at $p$ iff $X$ is a singleton. 
Proof. Let $(X, \mathcal{C})$ be $T_{0}^{\prime}$ at $p$ and $x, p \in X$. Since $(X, \mathcal{C})$ is $T_{0}^{\prime}$ at $p$ we have $\left\{x_{1} \in P\left(X \vee_{p} X\right)\right\}$. Assume that $\mathcal{C}^{2}$ is the product structure on $X^{2}$ and $W \in \mathcal{C}^{2}$, then we get $\nabla_{p}^{-1}(\{x\}) \cup i d_{p}^{-1}(W)=\left\{x_{1}\right\}$. Since $i d\left(x_{1}\right)=x_{1}$ $=(x, p) \in W$ and $W \in \mathcal{C}^{2}$, there exist $N, M \in \mathcal{C}$ such that $W=\pi_{1}^{-1}(N)$ $\cup \pi_{2}^{-1}(M)=(N \times X) \cup(X \times M)$. However, we find $\nabla_{p}^{-1}(\{x\})=\left\{x_{1}, x_{2}\right\}$, and $\left\{x_{1}\right\}=\nabla_{p}^{-1}(\{x\}) \cup A_{p}^{-1}(W)$ supset $\left\{x_{1}, x_{2}\right\}$, which is a contradiction. Therefore, $X=\{p\}$.

The other side of the implication is trivial.

Theorem 3.5 (cf. [11]). Let $(X, \mathcal{C})$ be a closure space and $p \in X$. $(X, \mathcal{C})$ is $T_{1}$ at $p$ iff $X=\{p\}$.

Proof. Suppose $(X, \mathcal{C})$ is $T_{1}$ at $p$ and $X \neq\{p\}$. Then there exists $x \in X$ with $x \neq p$. Since $(X, \mathcal{C})$ is $T_{1}$ at $p,\left\{x_{1}\right\}=\nabla_{p}^{-1}(\{x\}) \cup S_{p}^{-1}(W)$, where $W \in \mathcal{C}^{2}$ and $\mathcal{C}^{2}$ is the product structure on $X \times X$. Note that $\left\{x_{1}\right\}=\nabla_{p}^{-1}(\{x\}) \cup S_{p}^{-1}(W) \supset \nabla_{p}^{-1}(\{x\})=\left\{x_{1}, x_{2}\right\}$, which is a contradiction. Hence, $X=\{p\}$.

Conversely, let $X=\{p\}$. Note that the only closure structure on $X$ is given by $C=\{\emptyset,\{p\}\}=P(X)$. It follows from Definition 3.1 that $(X, \mathcal{C})$ is $T_{1}$ at $p$.

Remark 3.6. Let $U: \mathcal{E} \rightarrow \mathcal{B}$ be a topological functor. It was shown by Baran [9] that every indiscrete object in $\mathcal{E}$ is $\operatorname{Pre} \bar{T}_{2}$.

Theorem 3.7. Let $(X, \mathcal{C})$ be a closure space and $p \in X .(X, \mathcal{C})$ is Pre $\bar{T}_{2}$ at $p$ iff $(X, \mathcal{C})$ is an indiscrete closure space.

Proof. If $(X, \mathcal{C})$ is an indiscrete, then $(X, \mathcal{C})$ is $\operatorname{Pre} \bar{T}_{2}$ at $p$ since Remark 3.6. 
Assume that $(X, \mathcal{C})$ is $\operatorname{Pre} \bar{T}_{2}$ at $p$ and $X$ contains more than one element. In fact, $p \neq x$ and $x \in X$. Assume also that $N=\{p, x\} \subset X$ and the induced structure on $N$ is given by $\mathcal{C}_{N}=\{N \cap U: U \in \mathcal{C}\}$. We will show that $(N, \mathcal{C})$ is $\operatorname{Pr} e \bar{T}_{2}$ only when $\mathcal{C}_{N}$ is indiscrete. There are four cases.

Case 1. If $\mathcal{C}_{N}=\{N, \emptyset\}$, then $(X, \mathcal{C})$ is $\operatorname{Pre} \bar{T}_{2}$ at $p$ since Remark 3.6.

Case 2. If $\mathcal{C}_{N}=\{N, \emptyset,\{p\}\}$, then the product structure on $N^{2}$ is $\mathcal{C}_{N^{2}}=\left\{N^{2}, \emptyset,\{p\} \times N, N \times\{p\},(\{p\} \times N) \cup(N \times\{p\})\right\}=\left\{N^{2}, \emptyset,\{(p, p)\right.$, $(p, x)\},\{(p, p),(x, p)\},\{(p, p),(p, x),(x, p)\}\}$. Suppose that $W=N \times X \in \mathcal{C} N^{2}$ and $U=S_{p}^{-1}(W)=S_{p}^{-1}(N \times\{p\})=\left\{p_{1}\right\}$. However, there is no $W^{\prime}$ in $\mathcal{C}_{N^{2}}$ that gives the equality $S_{p}^{-1}(W)=A_{p}^{-1}\left(W^{\prime}\right)$. In fact, $S_{p}^{-1}\left(\mathcal{C}_{N^{2}}\right)=\left\{N \vee_{p} N, \emptyset,\left\{p_{1}, x_{2}\right\},\left\{p_{1}\right\}\right\}$. On the other hand, $A_{p}^{-1}\left(\mathcal{C}_{N^{2}}\right)=$ $\left\{N \vee_{p} N, \emptyset,\left\{p_{1}, x_{2}\right\},\left\{p_{1}, x_{1}\right\}\right\}$. Thus, $(X, \mathcal{C})$ is not $\operatorname{Pre} \bar{T}_{2}$ at $p$.

Case 3. If $\mathcal{C}_{N}=\{N, \emptyset,\{x\}\}$, then the product structure on $N^{2}$ is $\mathcal{C}_{N^{2}}=\left\{N^{2}, \emptyset,\{x\} \times N, N \times\{x\},(\{x\} \times N) \cup(N \times\{x\})\right\}=\left\{N^{2}, \emptyset,\{(x, p)\right.$, $(x, x)\},\{(x, x),(p, x)\},\{(x, p),(x, x),(p, x)\}\}$. Similar to Case 2, we obtain $S_{p}^{-1}\left(\mathcal{C}_{N^{2}}\right)=\left\{N \vee_{p} N, \emptyset,\left\{x_{1}\right\},\left\{x_{1}, x_{2}\right\}\right\}$ and $A_{p}^{-1}\left(\mathcal{C}_{N^{2}}\right)=\left\{N \vee_{p} N\right.$, $\left.\emptyset,\left\{x_{2}\right\},\left\{x_{1}\right\},\left\{x_{1}, x_{2}\right\}\right\}$. Therefore, $(X, \mathcal{C})$ is not $\operatorname{Pre} \bar{T}_{2}$ at $p$.

Case 4. If $\mathcal{C}_{N}=\{N, \emptyset,\{p\},\{x\}\}$, then the product structure on $N^{2}$ is $\mathcal{C}_{N^{2}}=\left\{N^{2}, \emptyset,\{p\} \times N, N \times\{p\},(\{p\} \times N) \cup(N \times\{p\}),\{x\} \times N, N \times\{x\}\right.$, $(\{x\} \times N) \cup(N \times\{x\})\}$. When we consider $W=N \times\{p\}$ we get $U=S_{p}^{-1}(W)=$ $S_{p}^{-1}(N \times p)=\left\{p_{1}\right\}$, but there is no $W^{\prime}$ in $\mathcal{C}_{N^{2}}$ which gives the equality $S_{p}^{-1}(N \times p)=A_{p}^{-1}\left(W^{\prime}\right)$. In fact, $A_{p}^{-1}\left(\mathcal{C}_{N^{2}}\right)=\left\{N \vee_{p} N, \emptyset,\left\{p_{1}, x_{2}\right\},\left\{p_{1}, x_{1}\right\}\right.$, $\left.\left\{x_{1}\right\},\left\{x_{2}\right\},\left\{x_{1}, x_{2}\right\}\right\}$. Hence, $S_{p}^{-1}\left(\mathcal{C}_{N^{2}}\right) \neq A_{p}^{-1}\left(\mathcal{C}_{N^{2}}\right)$. 
In general, let $X$ have at least two elements and $(X, \mathcal{C})$ is not indiscrete. Suppose also that $p, x \in X$ and $U \in \mathcal{C}$. If $U=\{x\}$ and $W=\pi_{2}^{-1}(U)=X \times U \in \mathcal{C}^{2}$, then $S_{p}^{-1}(W)=\left\{p_{1}\right\}$ but we obtain $A_{p}^{-1}(W)=$ $\left\{p_{1}, x_{1}, x_{2}, \ldots\right\}$. Hence $S_{p}^{-1}\left(\mathcal{C}^{2}\right) \neq A_{p}^{-1}\left(\mathcal{C}^{2}\right)$. In addition, if $U=\{x\}$ and $W=\pi_{2}^{-1}(U)=X \times U \in \mathcal{C}^{2}$, then $S_{p}^{-1}(W)=\left\{x_{1}, x_{2}\right\}$. However, $A_{p}^{-1}(W)=\left\{x_{1}\right\}$. Therefore $S_{p}^{-1}\left(\mathcal{C}^{2}\right) \neq A_{p}^{-1}\left(\mathcal{C}^{2}\right)$. We get similar results for the rest of the subsets of $X$ since they are unions of these sets. Therefore $(X, \mathcal{C})$ is not $\operatorname{Pre} \bar{T}_{2}$ at $p$ unless it is indiscrete.

Theorem 3.8. Let $(X, \mathcal{C})$ be a closure space and $p \in X .(X, \mathcal{C})$ is PreT $T_{2}^{\prime}$ at $p$ iff $(X, \mathcal{C})$ is an indiscrete closure space.

Proof. Let $(X, \mathcal{C})$ be $\operatorname{Pre}_{2}^{\prime}$ at $p$. It is trivial if $X=\emptyset$ or $X=\{p\}$. Assume that $X$ contains at least two elements and that $N=\{p, x\} \subset X$. The subspace structure on $N$ is given by $\mathcal{C}_{N}=\{N \cap U: U \in \mathcal{C}\}$ and the product structure on $N^{2}$ is $\mathcal{C}_{N^{2}}$. The final structure on $N \vee_{p} N$ obtained using the canonical injections $i_{1}$ and $i_{2}: N \rightarrow N \vee_{p} N$ is $\mathcal{C}_{\mathcal{N}}^{*}=\left\{U \subset N \vee_{p} N: i_{1}^{-1}(U) \in \mathcal{C}_{N}\right.$ and $\left.i_{2}^{-i}(U) \in \mathcal{C}_{N}\right\}$. We will find the condition that gives $S_{p}^{-1}\left(\mathcal{C}_{N^{2}}\right)=\mathcal{C}_{N}^{*}$. There are four cases.

Case 1. If $\mathcal{C}_{N}=\{N, \emptyset\}$, then clearly $S_{p}^{-1}\left(\mathcal{C}_{N^{2}}\right)=\left\{N \vee_{p} N, \emptyset\right\}=\mathcal{C}_{N}^{*}$. Hence, $(X, \mathcal{C})$ is $\operatorname{PreT}_{2}^{\prime}$ at $p$.

Case 2. If $\mathcal{C}_{N}=\{N, \emptyset,\{p\}\}$, then the product structure on $N^{2}$ is $\mathcal{C}_{N^{2}}=\left\{N^{2}, \emptyset,\{p\} \times N, N \times\{p\},(\{p\} \times N) \cup(N \times\{p\})\right\}$ and we get $S_{p}^{-1}\left(\mathcal{C}_{N^{2}}\right)=\left\{N \vee_{p} N, \emptyset,\left\{p_{1}, x_{2}\right\},\left\{p_{1}\right\}\right\}$. On the other hand, we find $\mathcal{C}_{N}^{*}=\left\{N \vee_{p} N, \emptyset,\left\{p_{1}, x_{2}\right\},\left\{p_{1}, x_{1}\right\},\left\{p_{1}\right\}\right\}$. Therefore, since $S_{p}^{-1}\left(\mathcal{C}_{N^{2}}\right)$ and $\mathcal{C}_{N}^{*}$ are different and $\left(N, \mathcal{C}_{N}\right)$ is not $\operatorname{PreT}_{2}^{\prime}$ at $p$. 
Case 3. If $\mathcal{C}_{N}=\{N, \emptyset\{x\}\}$, then the product structure on $N^{2}$ is $\mathcal{C}_{N^{2}}=\left\{N^{2}, \emptyset,\{x\} \times N, N \times\{x\},(\{x\} \times N) \cup(N \times\{x\})\right\}$ and we find $S_{p}^{-1}\left(\mathcal{C}_{N^{2}}\right)=\left\{N \vee_{p} N, \emptyset,\left\{x_{1}\right\},\left\{x_{1}, x_{2}\right\}\right\}$ However, the final structure is $\mathcal{C}_{N}^{*}=\left\{N \vee_{p} N, \emptyset,\left\{x_{1}\right\},\left\{x_{2}\right\}\right\}$. Thus $S_{p}^{-1}\left(\mathcal{C}_{N^{2}}\right) \neq \mathcal{C}_{\mathcal{N}}^{*}$ and $\left(N, \mathcal{C}_{N}\right)$ is not $\operatorname{Pre} T_{2}^{\prime}$ at $p$.

Case 4. If $\mathcal{C}_{N}=\{N, \emptyset,\{p\},\{x\}\}$, then the product structure on $N^{2}$ is $\mathcal{C}_{N^{2}}=\left\{N^{2}, \emptyset,\{p\} \times N, N \times\{p\},(\{p\} \times N) \cup(N \times\{p\}),\{x\} \times N, N \times\{x\}\right.$, $(\{x\} \times N) \cup(N \times\{x\})\}$. We have $S_{p}^{-1}\left(\mathcal{C}_{N^{2}}\right)=\left\{N \vee_{p} N, \emptyset,\left\{p_{1}, x_{2}\right\},\left\{p_{1}\right\},\left\{x_{1}\right\}\right.$, $\left.\left\{x_{1}, x_{2}\right\}\right\}$. On the other hand, we get $\mathcal{C}_{N}^{*}=\left\{N \vee_{p} N, \emptyset,\left\{p_{1}, x_{2}\right\}\right.$, $\left.\left\{p_{1}, x_{1}\right\},\left\{x_{1}\right\},\left\{x_{2}\right\},\left\{p_{1}\right\}\right\}$. Hence $S_{p}^{-1}\left(\mathcal{C}_{N^{2}}\right) \neq \mathcal{C}_{N}^{*}$, and so $\left(N, \mathcal{C}_{N}\right)$ is not $\operatorname{Pre} T_{2}^{\prime}$ at $p$.

In general, suppose that $X$ contains at least two elements. Then $\mathcal{C}^{2}$ is the product structure on $X^{2}$ and $\mathcal{C}^{*}=\left\{U \subset X \vee_{p} X: i_{1}^{-1}(U) \in \mathcal{C}\right.$ and $\left.i_{2}^{-1}(U) \in \mathcal{C}\right\}$ is the final structure on $X \vee_{p} X$ obtained by using canonical injections $i_{1}$ and $i_{2}: X \rightarrow X \vee_{p} X$. If $(X, \mathcal{C})$ is an indiscrete space, then since $S_{p}^{-1}\left(\mathcal{C}_{N^{2}}\right)=\left\{N \vee_{p} N, \emptyset\right\}=\mathcal{C}_{N}^{*}$, we easily conclude that $(X, \mathcal{C})$ is $\operatorname{Pre}_{2}^{\prime}$ at $p$.

Assume that $(X, \mathcal{C})$ is not indiscrete. Let $p, x \in X$ and $U \in \mathcal{C}$. If $U=\{p\}$, then we get $\mathcal{C}^{*}=\left\{X \vee_{p} X, \emptyset,\{p\} \vee_{p} X, X \vee_{p}\{p\},\{p\} \vee_{p}\{p\}\right\}$. However, $S_{p}^{-1}\left(\mathcal{C}^{2}\right)$ does not contain $\left\{x_{1}\right\}$ so $S_{p}^{-1}\left(\mathcal{C}^{2}\right) \neq \mathcal{C}^{*}$. On the other hand, if $U=\{x\}$, then we have $\mathcal{C}^{*}=\left\{X \vee_{p} X, \emptyset,\{x\} \vee_{p} X, X \vee_{p}\{x\}\right.$, $\left.\{x\} \vee_{p}\{x\}\right\}$. However, $S_{p}^{-1}\left(\mathcal{C}^{2}\right)=\left\{x_{1}, x_{2}\right\}$ and hence $S_{p}^{-1}\left(\mathcal{C}^{2}\right) \neq \mathcal{C}^{*}$. Since the rest of the subsets of $X$ are unions of these sets we obtain similar results. Hence $(X, \mathcal{C})$ is not $\operatorname{Pre} \bar{T}_{2}$ at $p$ unless it is indiscrete. 
Theorem 3.9. Let $(X, \mathcal{C})$ be a closure spaces. $(X, \mathcal{C})$ is $\bar{T}_{2}$ or $T_{2}^{\prime}$ at $p$ iff $X$ is a singleton or the empty set.

Proof. The proof is straightforward.

\section{Conclusion}

Various generalizations of separation properties for an arbitrary topological category over Set were given by Baran [5] in 1992 and recently, characterization of each of the separation properties $T_{i}$, $i=0,1,2$ at a point $p$ is given and relationships are examined in the topological category of Cauchy spaces by Kula [25]. In this paper, we found that separation properties $\bar{T}_{0}$ and $T_{0}^{\prime}$ at $p$ equivalent in the category of closure spaces. Similarly, we showed that $\operatorname{Pre} \bar{T}_{2}$ and $\operatorname{Pre} T_{2}^{\prime}$ at $p$ are equivalent in this category.

\section{References}

[1] J. Adámek, H. Herrlich and G. E. Strecker, Abstract and Concrete Categories, Wiley, New York, 1990.

[2] D. Aerts, Foundations of quantum physics: A general realistic and operational approach, Int. J. Theoret. Phys. 38(1) (1999), 289-358.

[3] D. Aerts, E. Colebunders, A. Van der Voorde and B. Van Steirteghem, State property systems and closure spaces: A study of categorical equivalence, Int. J. Theoret. Phys. 38(1) (1999), 359-385.

[4] G. Aumann, Kontaktrelationen, Bayer. Akad. Wiss. Math. -Nat. Kl. Sitzungsber (1970), 67-77.

[5] M. Baran, Separation properties, Indian J. Pure Appl. Math. 23 (1992), 333-341.

[6] M. Baran, The notion of closedness in topological categories, Comment. Math. Univ. Carolinae 34 (1993), 383-395.

[7] M. Baran, $T_{3}$ and $T_{4}$-objects in topological categories, Indian J. Pure Appl. Math. 29 (1998), 59-69.

[8] M. Baran, Compactness, perfectness, separation, minimality and closedness with respect to closure operators, Applied Categorical Structures 10 (2002), 403-415.

[9] M. Baran, PreT $T_{2}^{\prime}$-objects in topological categories, Applied Categorical Structures 17 (2009), 591-602. 
[10] M. Baran and H. Altindis, $T_{2}$-objects in topological categories, Acta Math. Hungar. 71(1-2) (1996), 41-48.

[11] M. Baran, D. Tokat and M. Kula, Connectedness and separation in the category of closure spaces, Filomat 24(2) (2010), 67-79.

[12] G. Birkhoff, The meaning of completeness, Ann. Math. 38 (1937), 57-60.

[13] G. Birkhoff, Lattice Theory, American Mathematical Society, Providence, Rhonde Island, 1940.

[14] E. Čech, On bicompact spaces, Ann. Math. 38 (1937), 823-844.

[15] V. Claes, E. Lowen-Colebunders and G. Sonck, Cartesian closed topological hull of the construct of closure spaces, Theory and Appl. of Categories (Electronic Journal) 8 (2001), 481-489.

[16] V. Claes and G. Sonck, The quasitopos hull of the construct of closure spaces, Appl. Gen. Topology 4 (2003), 15-24.

[17] D. Deses, E. Giuli and E. Lowen-Colebunders, On the complete objects in the category of $T_{0}$ closure spaces, Appl. Gen. Topology 4 (2003), 25-34.

[18] D. Dikranjan and E. Giuli, Closure operators I, Topology Appl. 27 (1987), 129-143.

[19] D. Dikranjan, E. Giuli and A. Tozzi, Topological categories and closure operators, Quaestiones Math. 11 (1988), 323-337.

[20] D. Dikranjan and W. Tholen, Categorical Structure of Closure Operators, with Applications to Topology, Algebra and Discrete Mathematics, Kluwer Academic Publishers, 1995.

[21] M. Erné, Lattice representations for categories of closure spaces, Categorical Topology, Sigma Series in Pure Mathematics 5, Heldermann Verlag Berlin, (1984), 197-222.

[22] B. Garner and R. Wille, Formal Concept Analysis, Springer Verlag, Berlin, 1998.

[23] E. Giuli, On classes of $T_{0}$ spaces admitting completions, Appl. Gen. Topology 4 (2003), 143-155.

[24] P. Hertz, Über Aximoensysteme für beliebige Satzsysteme, Teil I, Math. Ann. 87 (1922), 246-269.

[25] M. Kula, Separation properties at $p$ for the topological category of Cauchy spaces, Acta Math. Hungar. 136(1-2) (2011), 1-15.

[26] K. Kuratowski, Sur l'opération $\bar{A}$ de l'analysis Situs, Fund. Math. 3 (1922), 182-199.

[27] E. H. Moore, On a form of general analysis, with applications to linear differential and integral equations, in: Atti del IV Congress, Internationale di Mat., II, (Roma, 1909), pp. 98-114, 1909. 
[28] R. Pierce, Closure Spaces with Applications to Ring Theory, in: Lecture Notes in Math. 246, Springer, Berlin, 1972.

[29] F. Riesz, Stetigkeitsbegriff und abstrakte Mengenlehre, in: Atti del IV Congresso Internationale dei Mat., II, Roma, (1909), 18-24.

[30] A. Tarski, Remarques sur les notions fundamentales de la méthodologie des mathéma-tiques, Ann. Soc. Math. Polon. 7 (1929), 270-272. 